\title{
Determinants of Return to Home After Stroke: An Analysis Based on Familys' Views
}

\author{
HiroAki SAKURAi, RPT, MS ${ }^{1,2)}$, Yoshito SUGIURA, RPT, MS ${ }^{3)}$, TORU SigIURA, RPT ${ }^{2,4)}$, \\ KenJi IWATA, RPT ${ }^{2,4)}$, TAKAFUMI MATSUMOTO, MD ${ }^{4)}$, TETSURO KAWAMURA, $\mathrm{PhD}^{5}$, \\ TETSUO OKANISI, RPT, PhD ${ }^{1,2)}$, YOSHIKIYO KANADA, RPT, $\mathrm{PhD}^{1,2)}$ \\ ${ }^{1)}$ School of Health Sciences, Fujita Health University: 1-98 Dengakugakubo, Kutsukake, Toyoake, Aichi \\ 470-1192, Japan. TEL: +81 562-93-9000,FAX: +81 562-93-6817,E-mail: hsakurai@fujita-hu.ac.jp \\ ${ }^{2)}$ Graduate School of Health Sciences, Fujita Health University \\ 3) Department of Rehabilitation, Health Care Service Facility for The Aged, Tobahouwaen \\ 4) Medical Corporation Shoutokukai \\ ${ }^{5)}$ Suzuka University of Medical Science
}

\begin{abstract}
Purpose] To examine how the following factors would influence discharge to home: the family's view about the discharge destination at the time of the stroke patient's admission, the social background including the presence and sex of spouse and the number of family members, and the age of the patient. [Subjects] A total of 189 subjects with first time stroke were divided into 3 groups according to their families' views about discharge destination as follows: discharge to home group ( 96 subjects), discharge to facilities group (52 subjects), and undecided group (41 subjects). Each group was further divided into 2 sub-groups: discharge to home and discharge to facilities. [Methods] A multivariate logistic regression analysis was performed, considering the discharge destination as the response variables, and admission and discharge FIM scores of 18 items as explanatory variable. Also, in order to observe the influence of social background, comparisons by the presence and sex of spouse, by the number of family members, and by age were conducted. [Results] In a multivariate logistic regression analysis for all subjects at discharge, "eating" and "problem solving" were selected. In a comparison by age, a significant difference was recognized between those aged up to 79 years and those aged 80 years and older on the probability of discharge home. [Conclusion] The result suggests that eating and cognitive function play important roles in determining the discharge destination of stroke patients.
\end{abstract}

Key words: FIM, Discharge destination, Education

(This article was submitted Mar. 8, 2011, and was accepted Apr. 14, 2011)

\section{INTRODUCTION}

The number of deaths from stroke has been decreasing year by year, but there has been a trend toward increased morbidity. As a result, more and more elderly patients have been hospitalized in post-acute rehabilitation units (PARU), and the number of elderly patients having difficulty to return home is expected to increase. The rate of discharge from PARU to home was reported to be $70.9 \%$ at the All-Japan Post-Acute Rehabilitation Unit Conference held in February $2010^{1)}$. Preceding studies on factors influencing discharge to home have reported that independence in transferring, toileting, and getting on/off the toilet are predictive ADLrelated factors for patients returning home ${ }^{2)}$. Further, the presence of daytime and nighttime caregivers, a large number of family members, and the feasibility of temporary home-stay during hospitalization have also been demonstrated to be important contributing factors ${ }^{3-6}$.

The Functional Independence Measure (FIM), the most widely accepted ADL assessment measure instrument for stroke patients, has been used to investigate the determinants of discharge to home in relation to ADLrelated factors. The FIM has been proven to be highly reliable and valid ${ }^{7}$ and there are several studies focusing on discharge FIM scores ${ }^{2-6,8-13)}$. Among them, a study on the probability of discharge to home conducted by Toshima et al. reported that indicators for returning home include a discharge motor-FIM score of $70^{8)}$. Prediction of discharge destination has been investigated based on the amount of time from stroke onset to admission to PARU (onset-torehab time), predicted discharge FIM scores, the difference between admission and discharge FIM scores (FIM gain), and the rate of discharge to home ${ }^{9-11)}$. However, since there are some cases of discharge to care facilities despite a high FIM gain and to home despite a low FIM gain, it is necessary to investigate contributing factors in such cases. After examining determinants of discharge to home for patients with stroke using the FIM, we reported that three factors were determinants: toileting; length of stay; and age $^{12)}$. However, some reports have stated that not only 
Table 1. Property

\begin{tabular}{lcccccc}
\hline Desired by families & \multicolumn{2}{c}{$\begin{array}{c}\text { Discharge to home } \\
(\mathrm{n}=96)\end{array}$} & \multicolumn{2}{c}{$\begin{array}{c}\text { Discharge to facilities } \\
(\mathrm{N}=52)\end{array}$} & \multicolumn{2}{c}{$\begin{array}{c}\text { undecided } \\
(\mathrm{n}=41)\end{array}$} \\
\hline Observed discharge destination & $\begin{array}{c}\text { Home } \\
(\mathrm{n}=92)\end{array}$ & $\begin{array}{c}\text { facilities } \\
(\mathrm{n}=4)\end{array}$ & $\begin{array}{c}\text { Home } \\
(\mathrm{n}=11)\end{array}$ & $\begin{array}{c}\text { facilities } \\
(\mathrm{n}=41)\end{array}$ & $\begin{array}{c}\text { Home } \\
(\mathrm{n}=30)\end{array}$ & $\begin{array}{c}\text { facilities } \\
(\mathrm{n}=11)\end{array}$ \\
\hline age(years) & $75.1 \pm 9.6$ & $85.3 \pm 7.6$ & $77.5 \pm 7.9$ & $82.3 \pm 7.9$ & $80.8 \pm 6.9$ & $79.5 \pm 3.4$ \\
sex(males/females) & $40 / 52$ & $2 / 2$ & $7 / 4$ & $16 / 25$ & $12 / 18$ & $5 / 6$ \\
mean length of stay(days) & $79.1 \pm 37.6$ & $112.0 \pm 42.8$ & $105.6 \pm 36.5$ & $92.1 \pm 34.9$ & $103.7 \pm 30.8$ & $94.8 \pm 47.4$ \\
\hline
\end{tabular}

improvement in ADL but also relationships with family members at home are important factors for returning home ${ }^{6,14-16)}$, suggesting that improvement in ADL is not the sole determinant of discharge to home. In addition, since the number of studies on discharge to home considering family members' views about the discharge destination is limited, examination of the factors influencing discharge destination from viewpoints other than ADL is considered helpful foe the provision of more effective rehabilitation services.

The objective of this study was to examine how the following factors would influence discharge to home: the family's view about the discharge destination at the time of the stroke patient's admission (family's view), the social background including the presence and sex of spouse and the number of family members (living alone or living together), and the age of patient.

\section{SUBJECTS AND METHODS}

\section{Subjects}

Among stroke patients who were hospitalized and discharged during the period from June 1, 2008 to October 31,2010 , a total of 189 subjects ( 82 males and 107 females) who stayed in hospital for more than a month were included in the study after excluding cases of recurrence or complications. Their mean age was $78.2 \pm 9.3$ years, their mean onset-to-rehab time was $43.7 \pm 13.4$ days, and their mean length of stay was $89.9 \pm 37.7$ days. The number of patients discharged to home was 133 , and 56 were discharged to facilities. According to the type of disease, the number of patients with cerebral infarction was 132, 44 had that with cerebral hemorrhage, and 13 had subarachnoid hemorrhage.

As discussed in our previous paper "Determinants of Returning Home After Stroke: An Analysis Based on FIM Scores", social background factors also need to be examined. Therefore, subjects were divided into 3 groups according to their families views: family desired (FD) discharge to home group (96 subjects, $75.5 \pm 10.4$ years), FD discharge to facilities group (52 subjects, $81.7 \pm 8.0$ years), and undecided group (family undecided about discharge destination) (41 subjects, $80.4 \pm 5.9$ years). Each group was further divided into 2 sub-groups according to discharge destination (to home or to care facility), making 6 sub-groups in total. The breakdown was as follows: 92 were discharged to home $(75.1 \pm 10.3$ years $)$ and 4 were discharged to facilities $(85.3 \pm 7.6$ years $)$ from the FD discharge to home group; 11 were discharge to home (77.5 \pm 7.8 years $)$ and 41 were discharged to facilities $(82.7 \pm 7.7$ years) from the FD discharge to facilities group; and 30 were discharged to home $(80.5 \pm 6.9$ years $)$ and 11 were discharged to facilities $(79.5 \pm 3.4$ years $)$ from the undecided group (Table1).

\section{Methods}

The ADL of stroke patients was assessed on admission to the PARU and every other month until discharge using the FIM. It consists of 18 items related to self-care (eating, grooming, bathing, dressing upper body, dressing lower body, and toileting), sphincter control (bladder management and bowel management), transfers (bed/chair/wheelchair, toilet, and tub/shower), locomotion (walk/wheelchair and stairs), communication (comprehension and expression) and social cognition (social interaction, problem solving, and memory.) In order to examine determinants of discharge to home, age at admission and discharge, onset-to-rehab time, length of stay, and FIM score were compared between subjects discharged to home and discharged to facilities for all subjects, and undecided group. The Mann-Whitney U Test was used for statistics.

To examine determinants of discharge to home for each group, a multivariate logistic regression analysis was performed, considering the discharge destination (to home or to care facility) as the response variable, and admission and discharge FIM scores of 18 items for each sub-group as explanatory variables. In addition, in order to observe how social background influenced discharge to home, comparisons by the presence and sex of spouse, by number of family members, and by age were conducted using cross tabulation. The statistical software used in this study was PASW Statistics 18.0 for Windows. This study was conducted with the approval of the Ethical Review Boards of the facilities where patients hospitalized.

\section{RESULTS}

The total FIM scores at admission and at discharge for each sub-group were as follows: $69.9 \pm 29.0$ and $78.3 \pm 31.3$ for the 92 patients discharged to home from the FD discharge to home group (home-desired home sub-group); $45.0 \pm 14.3$ and $43.8 \pm 15.9$ for the 4 patients discharged to facilities from the FD discharge to home group (homedesired facility sub-group); $45.9 \pm 28.3$ and $57.4 \pm 31.5$ for the 11 patients discharged to home from the FD discharge to facilities group (facility-desired home sub-group); $33.8 \pm$ 17.1 and $40.2 \pm 25.7$ for the 41 patients discharged to 
Table 2. Comparison of the FIM scores between admission and discharge and discharge destination

\begin{tabular}{lcccc}
\hline & \multicolumn{2}{c}{ Admission } & \multicolumn{2}{c}{ Discharge } \\
\hline Observed discharge destination & Home $(\mathrm{n}=92)$ & Facilities $(\mathrm{n}=4)$ & Home $(\mathrm{n}=92)$ & Facilities(n=4) \\
\hline Self-care: eating & $5.4 \pm 1.6$ & $4.3 \pm 0.5^{*}$ & $5.8 \pm 1.5$ & $4.3 \pm 2.2^{*}$ \\
Self-care: grooming & $4.2 \pm 2.1$ & $3.3 \pm 1.7$ & $5.4 \pm 1.9$ & $3.0 \pm 1.6^{*}$ \\
Self-care: bathing & $3.0 \pm 2.1$ & $1.0 \pm 0.0^{*}$ & $4.2 \pm 2.1$ & $1.3 \pm 0.5^{*}$ \\
Self-care: dressing upper body & $4.3 \pm 2.1$ & $2.5 \pm 1.7$ & $5.5 \pm 2.0$ & $3.3 \pm 1.7^{*}$ \\
Self-care: dressing lower body & $3.8 \pm 2.3$ & $2.3 \pm 1.5$ & $5.2 \pm 2.2$ & $2.3 \pm 1.0^{*}$ \\
Self-care: toileting. & $4.0 \pm 2.2$ & $2.5 \pm 1.7$ & $5.2 \pm 1.8$ & $2.3 \pm 1.5^{*}$ \\
Sphincter control: bladder management & $4.2 \pm 2.5$ & $2.0 \pm 1.4$ & $5.1 \pm 2.3$ & $2.3 \pm 1.5^{*}$ \\
Sphincter control: bowel management & $4.2 \pm 2.5$ & $1.8 \pm 1.0$ & $5.3 \pm 2.0$ & $2.0 \pm 1.2^{*}$ \\
Transfers: toilet & $4.4 \pm 1.9$ & $3.5 \pm 1.3$ & $5.4 \pm 1.7$ & $3.3 \pm 1.5^{*}$ \\
Transfers: bed/chair/wheelchair & $4.5 \pm 1.8$ & $3.3 \pm 1.0$ & $5.6 \pm 1.6$ & $3.0 \pm 1.4^{*}$ \\
Transfers: tub/shower & $2.0 \pm 1.8$ & $1.0 \pm 0.0$ & $3.3 \pm 2.1$ & $1.0 \pm 0.0^{*}$ \\
Locomotion: walk/wheelchair & $2.8 \pm 2.3$ & $2.0 \pm 2.0$ & $4.3 \pm 2.4$ & $1.0 \pm 0.0^{*}$ \\
Locomotion: stairs & $1.3 \pm 1.0$ & $1.0 \pm 0.0$ & $2.3 \pm 2.0$ & $1.3 \pm 0.5$ \\
Communication: comprehension & $4.6 \pm 1.7$ & $3.3 \pm 1.0$ & $5.3 \pm 1.6$ & $3.3 \pm 1.0^{*}$ \\
Communication: expression & $4.8 \pm 1.6$ & $3.3 \pm 1.0$ & $5.3 \pm 1.6$ & $3.0 \pm 0.8^{*}$ \\
Social cognition: social interaction & $4.7 \pm 1.8$ & $3.3 \pm 1.9$ & $5.2 \pm 1.7$ & $2.3 \pm 0.5^{*}$ \\
Social cognition: problem solving & $3.9 \pm 1.7$ & $2.3 \pm 0.5$ & $4.5 \pm 1.7$ & $2.0 \pm 0.8^{*}$ \\
Social cognition: memory & $4.2 \pm 1.8$ & $2.8 \pm 0.5$ & $4.8 \pm 1.8$ & $2.8 \pm 0.5^{*}$ \\
\hline
\end{tabular}

$* \mathrm{p}<0.05$.

Table 3. Comparison of the FIM scores between admission and discharge and discharge destination

\begin{tabular}{lcccc}
\hline & \multicolumn{2}{c}{ Admission } & \multicolumn{2}{c}{ Discharge } \\
\hline Observed discharge destination & Home $(\mathrm{n}=11)$ & Facilities $(\mathrm{n}=41)$ & Home $(\mathrm{n}=11)$ & Facilities $(\mathrm{n}=41)$ \\
\hline Self-care: eating & $4.2 \pm 2.0$ & $2.8 \pm 2.0^{*}$ & $4.5 \pm 1.9$ & $3.0 \pm 2.3^{*}$ \\
Self-care: grooming & $3.3 \pm 2.0$ & $2.0 \pm 1.3^{*}$ & $4.0 \pm 2.0$ & $2.3 \pm 1.9^{*}$ \\
Self-care: bathing & $2.1 \pm 1.6$ & $1.3 \pm 0.7$ & $2.6 \pm 2.2$ & $1.6 \pm 1.2$ \\
Self-care: dressing upper body & $2.8 \pm 2.1$ & $1.7 \pm 1.3 *$ & $3.1 \pm 2.4$ & $2.5 \pm 2.0$ \\
Self-care: dressing lower body & $2.6 \pm 2.2$ & $1.6 \pm 1.3$ & $2.7 \pm 2.6$ & $2.2 \pm 2.0$ \\
Self-care: toileting. & $2.4 \pm 2.4$ & $1.7 \pm 1.1$ & $3.5 \pm 2.4$ & $2.2 \pm 1.9$ \\
Sphincter control: bladder management & $2.5 \pm 2.3$ & $1.8 \pm 1.5$ & $3.5 \pm 2.5$ & $2.0 \pm 1.9$ \\
Sphincter control: bowel management & $3.1 \pm 2.5$ & $1.9 \pm 1.6$ & $3.5 \pm 2.6$ & $2.0 \pm 2.0$ \\
Transfers: toilet & $3.0 \pm 2.1$ & $2.6 \pm 1.5$ & $3.9 \pm 2.2$ & $3.0 \pm 1.7$ \\
Transfers: bed/chair/wheelchair & $2.8 \pm 2.2$ & $2.3 \pm 1.6$ & $3.9 \pm 2.2$ & $2.6 \pm 1.9$ \\
Transfers: tub/shower & $2.0 \pm 1.7$ & $1.2 \pm 0.7$ & $2.2 \pm 2.0$ & $1.2 \pm 0.9$ \\
Locomotion: walk/wheelchair & $2.3 \pm 2.2$ & $1.2 \pm 0.8$ & $3.2 \pm 2.6$ & $1.8 \pm 1.7$ \\
Locomotion: stairs & $1.0 \pm 0.0$ & $1.0 \pm 0.3$ & $1.5 \pm 1.5$ & $1.2 \pm 0.9$ \\
Communication: comprehension & $2.5 \pm 1.3$ & $2.5 \pm 1.3$ & $3.5 \pm 1.4$ & $3.1 \pm 1.6$ \\
Communication: expression & $2.8 \pm 1.2$ & $2.4 \pm 1.3$ & $3.5 \pm 1.6$ & $3.1 \pm 1.5$ \\
Social cognition: social interaction & $2.5 \pm 1.8$ & $2.3 \pm 1.6$ & $3.2 \pm 1.8$ & $2.7 \pm 1.7$ \\
Social cognition: problem solving & $2.0 \pm 1.3$ & $1.9 \pm 1.3$ & $2.5 \pm 1.5$ & $1.9 \pm 1.4$ \\
Social cognition: memory & $2.0 \pm 1.0$ & $2.2 \pm 1.5$ & $3.0 \pm 1.8$ & $2.4 \pm 1.6$ \\
\hline
\end{tabular}

$* \mathrm{p}<0.05$

facilities patients from the FD discharge facilities group (facility -desired facility sub-group); $42.3 \pm 22.2$ and $57.7 \pm$ 27.1 for the 30 patients discharged to home from the undecided group (undecided home sub-group); and $31.6 \pm$ 16.3 and $42.5 \pm 26.8$ for the 11 patients discharged to facilities from the undecided group (undecided facility subgroup). The rate of discharge to home was $95.8 \%$ in the FD discharge to home group, $20.0 \%$ in the FD discharge facilities group, and $73.2 \%$ in undecided the group.

In the comparison of the FIM item scores of the FD discharge to home sub-groups, significant differences between the 2 sub-groups were observed admission in eating and bathing and also at discharge in all items except stairs (Table 2). In the comparison of the FIM item scores of the FD discharge to facilities sub-groups, significant differences between the 2 sub-groups were observed at admission in eating, grooming, and dressing upper body, and also on discharge in eating and bathing (Table 3 ). In the comparison of the FIM item scores of the undecided subgroups, significant differences were observed between these 2 sub-groups on admission in grooming and problem solving, and also at discharge in dressing upper body, expression, social interaction, and problem solving (Table 4).

In the multivariate logistic regression analysis considering the discharge destination of all 189 subjects as 
Table 4. Comparison of the FIM scores between admission and discharge and discharge destination

\begin{tabular}{lcccc}
\hline & \multicolumn{2}{c}{ Admission } & \multicolumn{2}{c}{ Discharge } \\
\hline Observed discharge destination & Home $(\mathrm{n}=30)$ & Facilities $(\mathrm{n}=11)$ & Home $(\mathrm{n}=30)$ & Facilities $(\mathrm{n}=11)$ \\
\hline Self-care: eating & $3.9 \pm 2.0$ & $2.5 \pm 2.1$ & $4.8 \pm 1.7$ & $3.1 \pm 2.5$ \\
Self-care: grooming & $2.3 \pm 1.4$ & $1.4 \pm 0.7^{*}$ & $3.2 \pm 1.8$ & $2.3 \pm 1.5$ \\
Self-care: bathing & $1.4 \pm 1.0$ & $1.3 \pm 0.6$ & $2.1 \pm 1.6$ & $1.7 \pm 1.3$ \\
Self-care: dressing upper body & $2.6 \pm 1.8$ & $1.8 \pm 1.5$ & $3.6 \pm 2.1$ & $2.2 \pm 2.2^{*}$ \\
Self-care: dressing lower body & $1.9 \pm 1.5$ & $1.6 \pm 1.5$ & $2.9 \pm 2.2$ & $2.4 \pm 2.4$ \\
Self-care: toileting. & $2.2 \pm 1.8$ & $1.7 \pm 1.5$ & $3.2 \pm 2$ & $2.3 \pm 1.6$ \\
Sphincter control: bladder management & $2.0 \pm 2.0$ & $1.5 \pm 1.5$ & $2.9 \pm 2.4$ & $2.5 \pm 2.3$ \\
Sphincter control: bowel management & $1.9 \pm 1.7$ & $1.5 \pm 1.5$ & $3.2 \pm 2.4$ & $2.4 \pm 2.2$ \\
Transfers: toilet & $2.6 \pm 1.6$ & $2.5 \pm 1.6$ & $4.2 \pm 1.7$ & $3.5 \pm 2.3$ \\
Transfers: bed/chair/wheelchair & $2.7 \pm 1.7$ & $2 \pm 1.7$ & $4.0 \pm 1.8$ & $3.3 \pm 2.2$ \\
Transfers: tub/shower & $1.1 \pm 0.7$ & $1.3 \pm 0.9$ & $1.6 \pm 1.3$ & $1.4 \pm 0.9$ \\
Locomotion: walk/wheelchair & $1.7 \pm 1.8$ & $1.8 \pm 1.8$ & $2.4 \pm 2.2$ & $2.3 \pm 2.2$ \\
Locomotion: stairs & $1.0 \pm 0.0$ & $1.0 \pm 0.0$ & $1.4 \pm 1.2$ & $1.3 \pm 0.9$ \\
Communication: comprehension & $2.8 \pm 1.3$ & $2.4 \pm 0.7$ & $3.8 \pm 1.5$ & $3.0 \pm 1.5$ \\
Communication: expression & $3.0 \pm 1.4$ & $2.4 \pm 1.1$ & $3.9 \pm 1.5$ & $2.7 \pm 1.3^{*}$ \\
Social cognition: social interaction & $2.8 \pm 1.5$ & $1.8 \pm 0.9$ & $3.7 \pm 1.7$ & $2.4 \pm 1.8^{*}$ \\
Social cognition: problem solving & $2.2 \pm 1.2$ & $1.3 \pm 0.5^{*}$ & $3.0 \pm 1.6$ & $1.6 \pm 1.2^{*}$ \\
Social cognition: memory & $2.3 \pm 1.3$ & $1.9 \pm 0.9$ & $3.2 \pm 1.7$ & $2.3 \pm 1.4$ \\
\hline * & & & &
\end{tabular}

$* \mathrm{p}<0.05$

the response variable, eating at discharge (odds ratio $=0.781$ ) and problem solving at discharge (odds ratio $=0.567$ ) were identified as significant explanatory variables (correct classification rate was $81.5 \%$ ). In the analysis considering the discharge destination of the 93 subjects in the FD discharge to facilities and undecided groups, eating at discharge (odds ratio $=0.680$ ) was identified as a significant explanatory variable (correct classification rate was $65.6 \%$ ). In the analysis considering the discharge destination of 148 subjects in the FD discharge to home and FD discharge to facilities groups with discharge destination as the objective variable, getting on/off the toilet at discharge (odds ratio $=0.555)$, comprehension at discharge (odds ratio $=1.792$ ), and problem solving at discharge (odds ratio $=0.449)$ were identified as significant explanatory variables (correct classification rate was $81.8 \%$ ). Among the FD discharge to home group, the FD discharge to facilities group, and the undecided group, no significant variable was identified in the FD discharge to home group; however, in the analysis considering the discharge destination of 52 subjects with the FD discharge to facilities group as the response variable, grooming at admission (odds ratio $=0.267$ ) and getting on/off toilet at admission (odds ratio $=2.324)$ were identified as significant explanatory variables (correct classification rate was $82.7 \%$ ). Likewise, for the 41 subjects in the undecided group, eating at admission (odds ratio $=0.655$ ) was identified as the significant explanatory variable (correct classification rate was $78.0 \%)$.

When comparing social background factors, which might be associated with the probability of discharge to home, there were no significant differences observed in the presence and sex of spouse and the number of family members (living alone or living together). However, a significant difference was seen in age, between those aged less than 80 years (98 subjects) and those aged 80 years or over (91 subjects) $(\mathrm{p}<0.05)$.

\section{DISCUSSION}

There have been many studies in which the FIM was used to examine the determinants of discharge to home $\mathrm{e}^{3-6,12-15)}$. Yoshida et al. reported that independence in toileting largely contributed to a $79 \%$ rate of returning home ${ }^{9}$. . Regarding the association between the discharge destination and toileting at discharge for stroke patients, Kondo et al. reported that the rate of discharge to home was $90 \%$ of patients who were independent in using portable toilets, but only $50 \%$ in the non-independent group ${ }^{17)}$. In our previous study, three factors, toileting, the length of stay, and age, were identified $^{12)}$ as significant variables when determinants of discharge to home for patients with poor ADL improvement were examined. Furthermore, in consideration that relationships with family at home may be more important factors for returning home, in this study, we examined how social background factors such as families views about discharge destination at the time patients were admitted would affect the probability of discharge to home. In the result, the factors associated with returning home in the FD discharge to home group were eating and bathing on admission, and items other than stair at discharge. In the FD discharge to home group, with good family support, $96 \%$ of the patients in this group returned home. However, there were 4 cases in this group who were discharged to care facilities because caring for the patients at home was not appropriate due to recurrence, aggravation of physical condition, gastrostomy etc. In the FD discharge to facilities group, there were significant differences observed between the 2 sub-groups in eating and glooming both at admission and at discharge. No dependency was noted in the difficulty 
level in performing FIM items ${ }^{18)}$, suggesting that the discharge destination could be care facilities if the patient needs supervision or assistance with ADL at admission. In addition, similar to subjects in the FD discharge to home group, many of the subjects discharged to facilities needed medical treatment and also the care burden at admission had not changed at discharge; therefore, it was considered difficult for them to be cared for at home and this resulted in their being discharged to a facility. In the undecided group, significant differences were observed between the 2 subgroups in grooming and problem solving at admission, and in dressing-upper body, expression, social interaction, and problem solving at discharge. For this group, it was considered that the influence of cognitive aspect was also a determinant of discharge destination besides ADL. Further, as a result of multivariate logistic regression analysis, it was found that getting on/off the toilet and problem solving were significant factors, similar to our previous research, in the FD discharge to home group and in the FD discharge to facilities group. However, in the undecided group, eating was also considered as another important factor.

In this study, eating was identified in all the groups as one of the determinants of discharge destination. Eating, often requires assistance with oral care and special diets, resulting in an overwhelming burden for family caregivers. Sugiura et al. reported that it was necessary to understand the health and physical conditions of patients, and predict and avoid the risk of aspiration pneumonia associated with anorexia/ dysphagia or poor nutrition ${ }^{19)}$, because the conditions of patients and their family may change over time after discharge to home. Furthermore, family caregivers have to provide medical treatments such as suctioning or oral care at home. All thoese factors, in turn, could make it difficult for patients to return home. Therefore, it is considered important to provide training to family members for such things as swallowing maneuvers, suctioning, oral brushing, and cooking during the hospital stay.

In the comparison of the social background factors which were considered as possible factors influencing the probability of discharge to home, there was no significant difference observed in the presence and sex of spouse and number of family members. However, some reports have suggested that not only the improvement in ADL but also the relationship with family was more important as a factor associated with discharge to home $\mathrm{e}^{6,14-16)}$, Therefore, we considered it necessary to further examine the relationship with key persons other than the spouse. In this study, in the comparison by age, the results indicate that 80 years old may be the age above which the probability of being discharged to home can be determined. However, Ikeda et al. reported that for those who are 75 years or older, 5 years younger than the 80 years, age may become a determinant of discharge to home ${ }^{20)}$. In both cases, it was indicated that age played an important role in determining the discharge destination of patients with stroke.

Since the death rate from stroke has been declining, discharging elderly patients from PARU to home has become an important issue. This study was designed to be of help in providing physical therapy that addresses the concerns that patients' families have with regard to caring, in addition to ADL. In future we would like to conduct a study on psychological factors of family members, including motivation and burden of care, which are necessary to be considered in families' desire for discharge to home.

\section{REFERENCES}

1) National recovery period Riha Ward Liaison Council: Survey report on the present and future of recovery rehabilitation ward. Tokyo: 2010, p32.

2) Umemoto Y: Factors that affect recovery Riha Ward home return - FIM From study - Aichi Occupational Therapy, 2008, 16: 33-36.

3) Asakawa I, Imura $S$, Usuda $S$, et al.: Factors influencing post rehabilitation outcomes of cerebrovascular accident patients with lower than 90 points on the FIM in a convalescence rehabilitation ward. Phys Ther Sci, 2008, 23: 545 550.

4) Goto K: The examination of the factor about the home return of patients with cerebrovascular disorder in the convalescent rehabilitation ward. Aichi physical therapy official journal of a scientific society, 2008, 20: 42-43.

5) Niki R: A medical social condition for patients with stroke to leave the hospital at home. Gen Rehab, 1983, 11: 895-899.

6) Uematsu K: A conditions for aged stroke patients in order to desired to discharge home. Rehab Med, 2002, 39: 396-402.

7) Mizuno K, Ota T: Outcome metrics in rehabilitation - FIM, Barthel Index -. Clin Rehabil, 2005, 14: 174-179.

8) Toshima M, Nishitani M, Hagiwara R: Factors affecting length of stay and discharge destination in patients with acute ischemic stoke. Rehabil Med, 2001, 38: 268-276.

9) Yoshida K, Takada T: Evaluation of outcome in stroke patients by convalescence rehabilitation start time using the motor FIM. Stroke, 2006, 28: 396-402.

10) Yavuzer G: Rehabilitation of stroke patient: Am J Phys Med Rehabil, 2001, 80: 250-255.

11) Inouye M: Predicting models of outcome statified by age after first stroke rehabilitation. Am J Phys Med Rehabil, 2001, 80: 586-591.

12) Sakurai H, Tsujimura T, Sugiura $Y$ : Determinants of returning home after stroke: an analysis based on FIM scores. J Phys Ther Sci, 23, 2011.

13) Goto $S$, Kitagawa A, Taka K: Prediction of functional independence measure in a sub-acute cerebrovascular accident patient at the time of a dischargeexternal validity and comparison with the targeted value by the staff. Gen Rehabil, 2008, 36: 275-281.

14) Yagutchi H, Yaguchi M: Convalescent rehabilitation outcome of stroke patients 90 years old and older. J Clin Rehabil, 2010, 19: 195-198.

15) Nagai S, Sonoda S, Kakehi A: The relationship between intensity of stroke rehabilitation and outcome. Sogo Rehabilitation, 2009, 37: 547-553.

16) Nishida $Y$, Ohtsuka $Y$, Hara $T$ ed: Comparison of the reqirecare level by longterm care insurance service using circumstance for day care user. Phys Ther Sci, 2004, 19: 37-41.

17) Kondo K: Evidence of the medical reform and rehabilitation medicine. Rehabil Med, 2006, 43: 651-657.

18) Sakurai H, Saitoh E, Watanabe A: Analysis of functional independence measure item in acute stroke patient. Bulletin of the Fujita Medical Society, 2006, 30: 89-94.

19) Sugiura $Y$, Sakurai $H$, Kanada $Y$ : Approach for dysphagia in the elderly living at home. Geriat Med, 2009, 47: 613-616.

20) Takashi I, Satoko Y, Ikejima K: Predictors of Desired to discharge home after Acute Stroke in Bedside Evaluation: Does Age Affect the Factors? Phys Ther Sci, 2010, 25: 23-27. 